\title{
Profiling the nucleobase and structure selectivity of anticancer drugs and other DNA alkylating agents by RNA sequencing
}

\begin{abstract}
Basilius Sauter, ${ }^{[a]}$ and Dennis Gillingham*[a] uncharacterized alkylating agent trimethylsilyl diazomethane.
\end{abstract}

transcriptional modifications influence sequencing datasets has recently been explored. ${ }^{[12]}$ The techniques most similar in workflow to what we describe here are SHAPE sequencing and other types of high-throughput chemical RNA probing. ${ }^{[13]}$ Whereas, however, chemical probing looks at characterizing RNA secondary structure or protein binding sites, we hope to offer a profile of the chemicals themselves: the types of bases they react with and in what ratios, as well as whether they prefer specific consensus sequences or specific types of structures.

A Key idea: RNA sequencing can profile the selectivity of alkylating agents

DNA alkylating agents are extremely toxic molecules, and yet they remain a cornerstone of chemotherapy. ${ }^{[1]}$ In glioblastoma multiforme, for example, the alkylating agent temozolomide $(T M Z)^{[2]}$ is the only approved drug. One of the world's first anticancer agents was a sulfur mustard, ${ }^{[3]}$ but many other DNA alkylating molecules ${ }^{[4]}$ have been discovered either by sifting through natural products that bear electrophiles, or by focused medicinal chemistry efforts on electrophilic scaffolds with a known predilection for nucleic acid modification. ${ }^{[5]}$ While alkylating DNA to cause apoptosis is their main mode of action, these alkylating agents are indiscriminately reactive, leading to many side effects. One of the off-target substrates is the more abundant RNA, but whether this contributes to toxicity is unknown. Damage to mRNA is mostly short-lived but can lead to longer-lasting effects if they cause errors during translation, ${ }^{[6]}$ or if the damage causes a catalytic gain-of-function. ${ }^{[6]}$ With these findings and the discovery of RNA repair proteins, ${ }^{[7]}$ the significance of RNA damage needs to be re-evaluated. ${ }^{[6 a, 6 b, 8]}$ Since all of the alkylating drugs on the market were discovered before the advent of next generation sequencing (NGS), studies of their chemical reactivity on nucleic acids were necessarily serial, limiting analyses to a small set of synthetic oligodeoxynucleotides or to low-throughput biochemical assays such as ligation-mediated PCR..$^{[9]}$ Nucleobase selectivity was typically characterized by reactions with mononucleotides, which cannot report on sequence context or structure specificity. NGS would offer a more nuanced and statistically powerful profiling of alkylating agents, but this has gone largely untested. A couple of recent NGS approaches that look at sites of cisplatin reactivity ${ }^{[10]}$ in both DNA and RNA are the lone exceptions. Other reports have looked at whether there might be natural or designed RNA aptamer sequences that can predispose an RNA to alkylation by organic electrophiles. ${ }^{[1]}$ How natural post-

[a] Basilius Sauter, Prof. Dr. Dennis Gillingham

Department of Chemistry

University of Basel

St. Johanns-Ring 19

4056 Basel

E-mail: dennis.gillingham@unibas.ch

Supporting information for this article is given via a link at the end of the document.

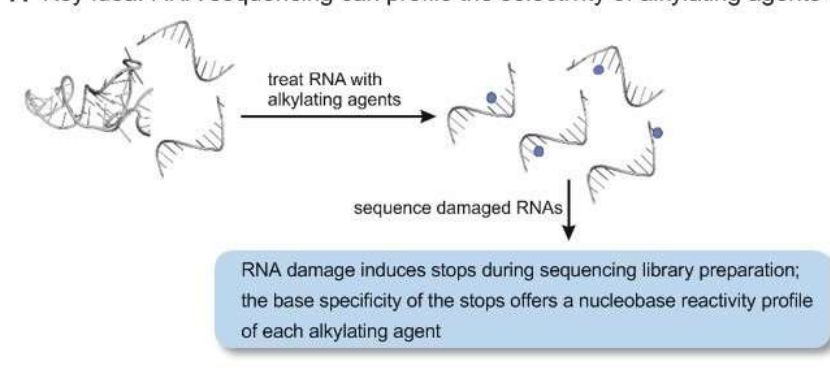

B Alkylating agents profiled by RNA sequencing

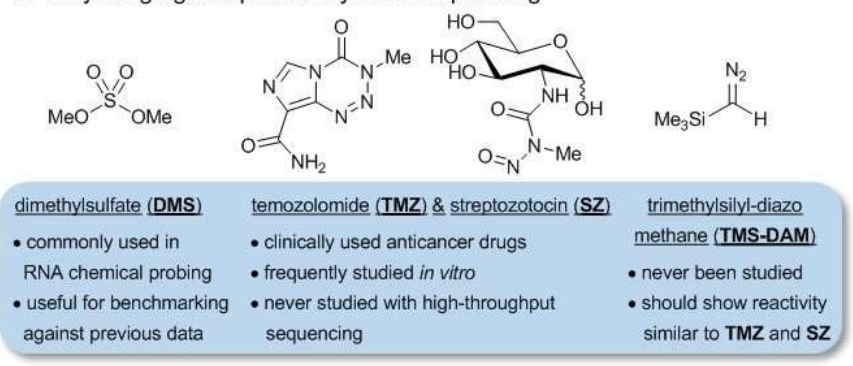

Figure 1. Profiling the selectivity of nucleic acid damaging agents by RNA sequencing

To develop the protocol, we choose to characterize two known drugs that operate by a similar mechanism and have a wellestablished chemical reactivity profile in DNA. TMZ ${ }^{[2 b]}$ and streptozotocin (SZ) (see Figure 1B for structures) ${ }^{[14]}$ are both purported to generate methyldiazonium upon nucleophilic activation and this powerful electrophile ${ }^{[15]}$ then preferentially targets $\mathrm{G}$, modifying both its $\mathrm{N} 7$ and $\mathrm{O}^{6}$ positions. ${ }^{[16]}$ After establishing the protocol with these two molecules, we implemented the method to profile the reactivity of trimethylsilyldiazomethane (TMS-DAM) and dimethylsulfate (DMS). The reactivity of TMS-DAM with DNA and RNA is unstudied, but in principle should show similar reactivity as TMZ and $\mathbf{S Z}$ since it also generates a methyldiazonium in protic solvents. ${ }^{[17]}$ DMS, on the other hand, is frequently used as a reagent in chemical RNA probing and in fact was one of the key reagents for the original Maxam-Gilbert sequencing. ${ }^{[18]}$ Indeed, our sequencing data jibes with the observed chemical reactivity profile in Maxam-Gilbert sequencing. The advantage of NGS is 
that new alkylating agents with unknown reactivity can be quickly profiled across a broad set of sequences in a single experiment. We started by developing the sequencing method and data analysis pipeline to identify sites of alkylation. Over the past few years classical RNA chemical profiling methods have been adapted for whole transcriptome NGS. ${ }^{[13]}$ Data analysis approaches vary, but the basic concept in these techniques is the same: RNAs are chemically modified and then reverse transcribed (RT) to complementary DNAs (cDNAs). The chemically modified positions prematurely halt RT or cause mutations during read-through; modifications in the original RNA can then be inferred from premature stops or mutations in the sequencing reads. While these techniques were developed to study epigenetics and RNA structure, our goal was to adapt them for broad characterization of the chemo- and site-selectivity of nucleic acid alkylating agents.

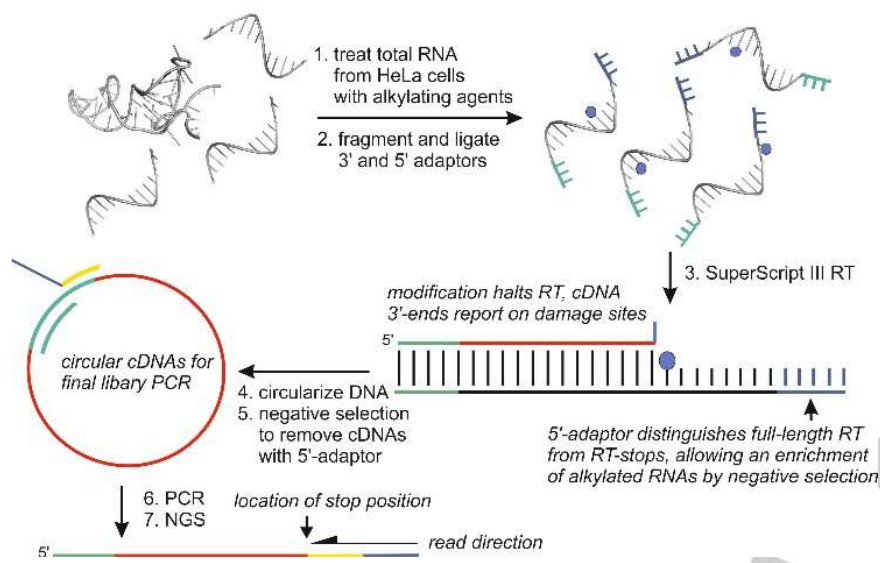

Figure 2. Sequencing library preparation protocol for profiling RNA damage selectivity.

Our protocol is similar to those used in previous reports in RNA structure based profiling, ${ }^{[13]}$ but most closely resembles the ModSeq protocol reported by the McManus lab. ${ }^{[13 c]}$ The biggest changes we've made to the McManus protocol are that we use STAR $^{[19]}$ for genome alignment and that we use an alkaline fragmentation protocol (more on this below). In brief, we began by treating HeLa cells or HeLa whole transcriptome isolates with a variety of alkylating agents at high concentration (Figure 2, step 1). The resulting RNA pools were then fragmented, and adaptors were ligated at both ends (step 2). The 3' adaptor is a universal PCR primer, while the $5^{\prime}$ adaptor is meant to serve as a stopmarker downstream in the bioinformatics analysis. An enrichment step with magnetic beads bearing a complement to the 5' adaptor insures that only successful ligation products proceed to the next step. RT then creates cDNAs of two types; RTs that stop prematurely will give cDNAs that lack a complement to the originally ligated 5' stop-marker, while those that copy normally will have this sequence at their 3' ends. The cDNAs are then circularized and the sequences that bear the stop-marker adaptor are removed by a magnetic bead negative selection (the beads contain an oligonucleotide complementary to the cDNA stop marker). The remaining CDNAs are then PCR amplified with primers that bear the Illumina sequences, purified with AMPure beads to minimize empty primer reads and then submitted for NGS sequencing.
As already mentioned, the biggest difference between our method and SHAPE sequencing is that we use alkaline fragmentation. Although $\mathrm{ZnCl}_{2}$ fragmentation was also an option, ${ }^{[13 d]}$ we opted for the alkaline method for chemical profiling since it reports on total modification (since N7G alkylations are depurinated under alkaline conditions and hence turn up as stops in the data), while the $\mathrm{ZnCl}_{2}$ method (which does not efficiently depurinate) reports more on the modifications in the Watson-Crick face. Before doing any data filtering we first wanted to analyze whether the drug treated samples showed any consistent changes in stop positions across all stops. Indeed, in the treated samples we saw a substantial increase in the number of stops at guanine residues. We then reanalyzed the stops after applying a significance test filtering (Cochran-Mantel-Haenszel testing according to the method previously described). ${ }^{[13 c]}$ We first analyzed the stop-signal for the four alkylating agents shown in Figure $1 \mathrm{~B}$ both in vitro and in live cells. For all but DMS this is the first time that these DNA alkylating agents have been characterized with NGS. As expected, they all showed a preference for guanine alkylation (signals are determined by comparison with matched DMSO controls). None of these purportedly unspecific alkylating agents show a sequence logo preference (see the ESI), consistent with their generating a potent but unselective alkylating agent. The alkylating profiles in live cells match closely those seen with transcriptome isolates except for TMZ. TMZ demonstrated extremely poor solubility in the culture medium, we believe this leads to a low effective treatment concentration and hence the stop-signals with TMZ are indistinguishable from the untreated controls. We also independently measured each base's propensity for RNA alkylation with DMS by reacting the monoribonucleotides with DMS and then examining the product ratios by UPLC-MS; this method gives conversions of $86,12,6$, and 0 for $G, A, C$, and $U$ respectively (see ESI figure $\mathrm{S} 11$ for data), exactly as the reactivity order predicted by sequencing based profiling in vitro (see DMS signals in panels $B$ of Figure 3). These profiles are also consistent with earlier work on DNA modification ${ }^{[16,20]}$ with DMS, which suggests that the order of reactivity should be $G>A>C>T$ in an approximately $78: 20: 2: 0$ ratio. 
A total stop counts from raw sequencing data
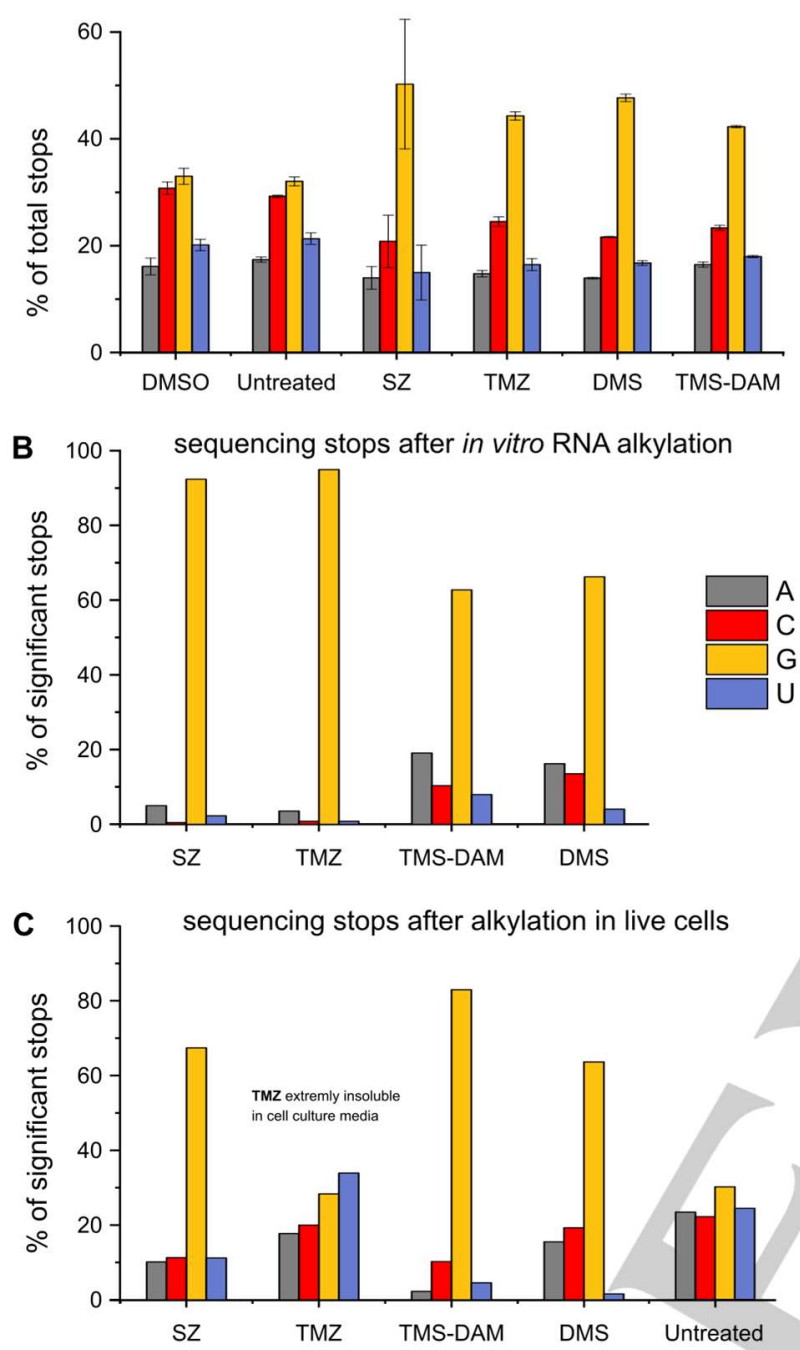

Figure 3. (A) Crude sequencing data indicates a significant preference for guanine stops in comparison to untreated controls; (B) Applying the profiling sequencing workflow on the in vitro data delivers stops that show a strong preference for G; (C) Alkylation reactions in cell culture analysed by the chemical profiling method show consistent profiles except $T M Z$, which is highly insoluble in the culture medium (note: analyses in B and C excluded ribosomal RNA (rRNA) since they are the most populous and any selectivity inherent to rRNA would therefore dominate the data).

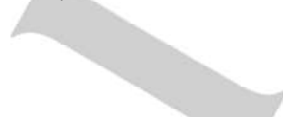

In structural probing tertiary structures are approximated by calculating a reactivity of each nucleobase position. ${ }^{10 \mathrm{~d}}$ In Figure 4 we focus on the 18S rRNA to examine in detail structural specificities of alkylation. In Figure 4A we see that TMS-DAM would be a poor structural probing reagent because it can target both double and single-stranded regions. On the other hand, if we look at the $A$ and $C$ stops for DMS, we see a strong preference for single stranded regions, a feature that makes DMS an excellent chemical probing reagent. The more broad reactivity of TMS-DAM, however, could be more advantageous for probing intermolecular interactions. Indeed if we look at the live cell results for both TMS-DAM and DMS we see that modification at double- stranded regions is completely lost. Taken together, these analyses validate that NGS is an effective way to profile the structural selectivity of DNA and RNA damaging agents. As shown in Figure 4B, only guanine is alkylated in double-stranded regions in 18S rRNA in the live cell experiments. This unexpected observation could stem from several factors; the most interesting would be that this reactivity difference is reporting on tertiary or quaternary interactions of the $18 \mathrm{~S}$ rRNA. Going forward we would like to increase sequencing depths so that we can search for putative sites of intermolecular interactions with statistical confidence, and then compare putative interactions with other recently described probing methods. ${ }^{[13 f, 21]}$

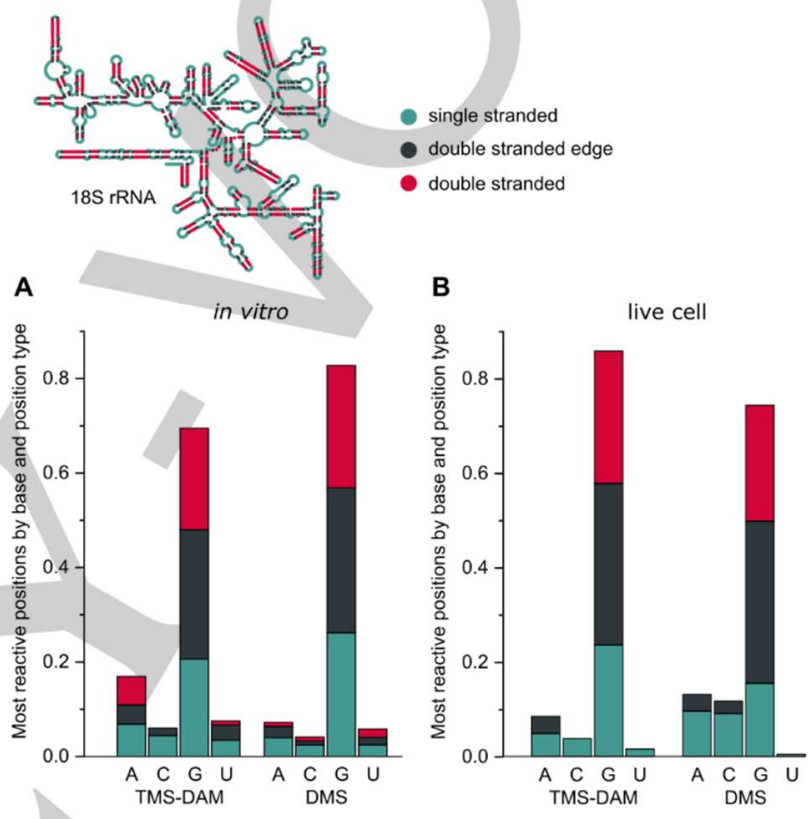

Figure 4. Selectivity of alkylation for different structural motifs in 18s rRNA in vitro (A) and in live cells (B).

A disadvantage of the technique is that it is blind to modifications that cause neither RT stops nor frequent mutations. TMS-DAM offers an example of this shortcoming. Alkylation reactions with diazo electrophiles require a free proton to activate the diazo to a highly electrophilic diazonium. ${ }^{[22]}$ Guanine and uracil have similar $\mathrm{pK}_{\mathrm{a}} \mathrm{s}$ and they are the first ionisable protons in oligoribonucleotides, ${ }^{[23]}$ which should give them similar reactivity with TMS-DAM. We tested the reactivity of all four RNA bases with TMS-DAM as mononucleosides or in HeLa total transcriptome RNA (Figure 5A). In both cases the major product was the $m^{3} U$ alkylation product followed by $m^{6} G$ and $m^{1} G$. The Kool lab has recently shown that $\mathrm{m}^{3} \mathrm{U}$ is relatively well-tolerated during RT, making it difficult to detect in NGS datasets. ${ }^{[12]}$ Our data is consistent with this conclusion since although there is substantial uridine modification according to chemical analysis, the NGS data show predominately guanine alkylation. With this in mind, a protocol for characterizing new alkylating agents would be to examine the in vitro specificity in mononucleotides and then use NGS to look for sequence or structural selectivity across many different sequences. 

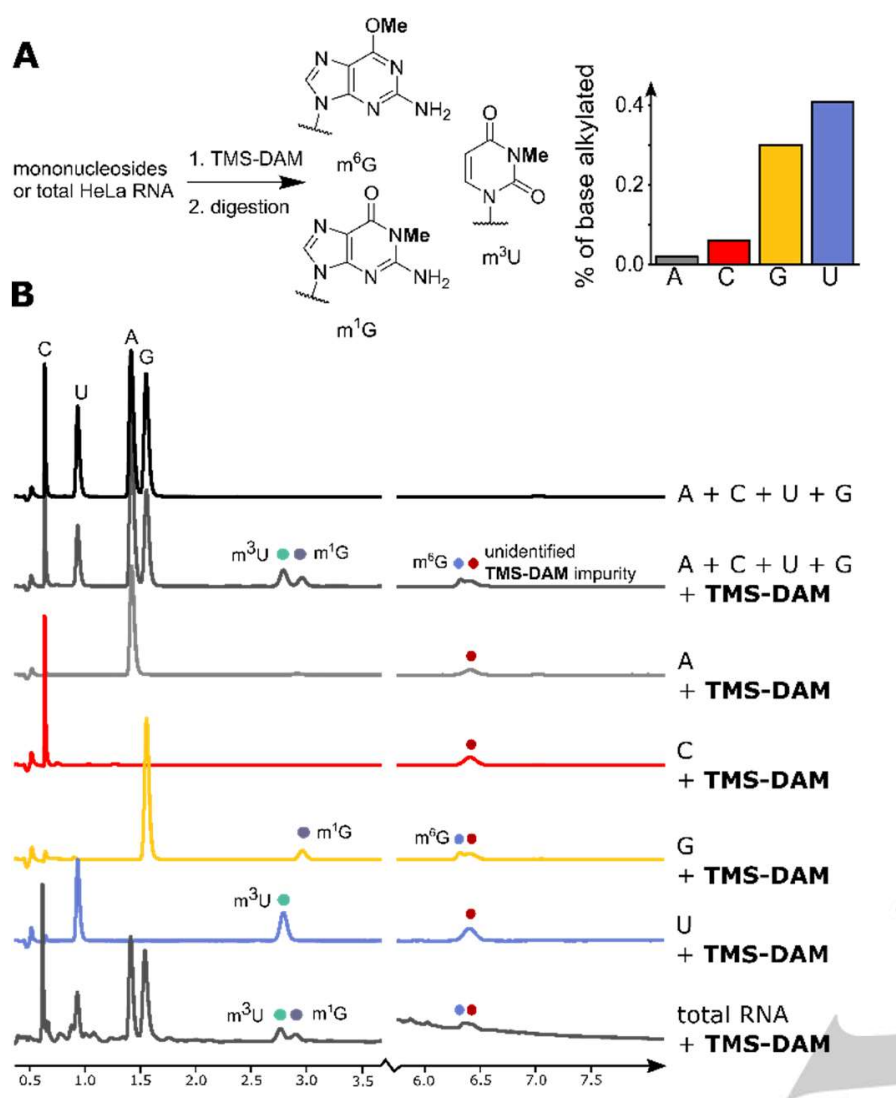

Figure 5. A. Alkylation reactions with TMS-DAM were carried out on both monoribonucleosides and total HeLa RNA. In the case of total RNA a digestion was carried out to generate monoribonucleosides for analysis; the bar graph shows the relative reactivity of each base; $\mathbf{B}$. Crude HPLC traces show that A and $C$ are not modified, while $G$ gives two products $\left(m^{1} G\right.$ and $\left.m^{6} G\right)$ and $U$ gives a single product $\left(\mathrm{m}^{3} \mathrm{U}\right)$.

We've shown that RNA sequencing can be used to chemically profile alkylating anti-cancer agents or any other molecule that can covalently modify RNA and gives RT stops or mutations during cDNA synthesis. To develop the method, we first chose electrophiles with a well-established reactivity profile in DNA, and then profiled a substrate that has never been analysed in RNA alkylation (TMS-DAM) to show how the technique could be used in practice. To get a high enough signal-to-noise ratio, we needed to use high drug concentration. While this is an effective strategy for chemical profiling and RNA probing, it might obscure valuable additional information such as whether there are any sequence or transcript preferences. In the future we will include an enrichment step in reactions with modified electrophiles, allowing us to use reduce drug concentrations to levels that would not oversaturate any inherent drug selectivity and will help us identify whether alkylating drugs damage specific transcripts or consensus sequences. Already in its present form, however, the technique is an efficient way to profile the nucleobase and tertiary structural selectivity of DNA and RNA damaging agents.

\section{Acknowledgements}

Prof. Mihaela Zavolan and Dr. Nitish Mittal are gratefully acknowledged for help in establishing the sequencing protocol and tips on the bioinformatics analysis. Calculations were performed at sciCORE (https://scicore.unibas.ch/); the scientific computing core facility of the University of Basel.

Keywords: RNA damage $\cdot$ alkylating chemotherapeutics • copper catalysis • diazo chemistry $\bullet$ RNA sequencing

[1] A. P. Francisco, M. d. J. Perry, R. Moreira, E. Mendes, in Anticancer Therapeutics, John Wiley \& Sons, Ltd, 2008, pp. 133-158.

[2] (a) Z. Jihong, F. G. S. Malcolm, D. B. Tracey, Curr. Mol. Pharm. 2012, 5 102-114; (b) E. Newlands, M. Stevens, S. Wedge, R. Wheelhouse, C. Brock, Cancer Treat. Rev. 1997, 23, 35-61.

[3] F. E. Adair, H. J. Bagg, Ann. Surg. 1931, 93, 190-199.

[4] A. Haddow, G. A. R. Kon, W. C. J. Ross, Nature 1948, 162, 824

[5] (a) W. C. Tse, D. L. Boger, Chem. Biol. 2004, 11, 1607-1617; (b) S. E Wolkenberg, D. L. Boger, Chem. Rev. 2002, 102, 2477-2496.

[6] (a) C. L. Simms, H. S. Zaher, Cell. Mol. Life Sci. 2016, 73, 3639-3653 (b) B. H. Hudson, H. S. Zaher, RNA 2015, 21, 1648-1659; (c) J. R Brickner, J. M. Soll, P. M. Lombardi, C. B. Vågbø, M. C. Mudge, C Oyeniran, R. Rabe, J. Jackson, M. E. Sullender, E. Blazosky, A. K. Byrum, Y. Zhao, M. A. Corbett, J. Gécz, M. Field, A. Vindigni, G Slupphaug, C. Wolberger, N. Mosammaparast, Nature 2017, 551, 389; (d) H. S. Pettersen, T. Visnes, C. B. Vågbø, E. K. Svaasand, B. Doseth, G. Slupphaug, B. Kavli, H. E. Krokan, Nucleic Acids Res. 2011, 39, 8430 8444; (e) J.-X. Wang, J. Gao, S.-L. Ding, K. Wang, J.-Q. Jiao, Y. Wang, T. Sun, L.-Y. Zhou, B. Long, X.-J. Zhang, Q. Li, J.-P. Liu, C. Feng, J. Liu, Y. Gong, Z. Zhou, P.-F. Li, Mol. Cell 2015, 59, 50-61.

[7] (a) G. Zheng, John A. Dahl, Y. Niu, P. Fedorcsak, C.-M. Huang Charles J. Li, Cathrine B. Vågbø, Y. Shi, W.-L. Wang, S.-H. Song, Z. Lu, Ralph P. G. Bosmans, Q. Dai, Y.-J. Hao, X. Yang, W.-M. Zhao, W.-M. Tong, X.-J. Wang, F. Bogdan, K. Furu, Y. Fu, G. Jia, X. Zhao, J. Liu, Hans E. Krokan, A. Klungland, Y.-G. Yang, C. He, Mol. Cell 2013, 49, 18-29; (b) M. Westbye, E. Feyzi, P. Aas, C. Vågbø, V. Talstad, B. Kavli, L. Hagen, O. Sundheim, M. Akbari, N.-B. Liabakk, G. Slupphaug, M. Otterlei, H. Krokan, J. Biol. Chem. 2008, 283, 25046-25056; (c) F Drabløs, E. Feyzi, P. A. Aas, C. B. Vaagbø, B. Kavli, M. S. Bratlie, J. Peña-Diaz, M. Otterlei, G. Slupphaug, H. E. Krokan, DNA Repair 2004 3, 1389-1407; (d) P. Aas, M. Otterlei, P. Falnes, C. Vågbø, F. Skorpen, M. Akbari, O. Sundheim, M. Bjørås, G. Slupphaug, E. Seeberg, H. Krokan, Nature 2003, 421, 859-863.

[8] C. L. Simms, B. H. Hudson, J. W. Mosior, A. S. Rangwala, H. S. Zaher Cell Reports 2014, 9, 1256-1264

[9] G. P. Pfeifer, R. Drouin, G. P. Holmquist, Mutat. Res. 1993, 288, 39-46.

[10] (a) X. Shu, X. Xiong, J. Song, C. He, C. Yi, Angew. Chem. Int. Ed. 2016 55, 14246-14249; (b) J. Hu, J. D. Lieb, A. Sancar, S. Adar, Proc. Natl. Acad. Sci. USA 2016, 113, 11507-11512; (c) K. Plakos, V. J. DeRose, Chem. Commun. 2017, 53, 12746-12749.

[11] (a) R. I. McDonald, J. P. Guilinger, S. Mukherji, E. A. Curtis, W. I. Lee, D. R. Liu, Nat. Chem. Biol. 2014, 10, 1049-1054; (b) A. K. Sharma, J. J. Plant, A. E. Rangel, K. N. Meek, A. J. Anamisis, J. Hollien, J. M. Heemstra, ACS Chem. Biol. 2014, 9, 1680-1684.

[12] A. M. Kietrys, W. A. Velema, E. T. Kool, J. Am. Chem. Soc. 2017, 139, 17074-17081.

[13] (a) D. Loughrey, K. E. Watters, A. H. Settle, J. B. Lucks, Nucleic Acids Res. 2014, 42, 000; (b) J. B. Lucks, S. A. Mortimer, C. Trapnell, S. Luo, S. Aviran, G. P. Schroth, L. Pachter, J. A. Doudna, A. P. Arkin, Proc. Natl. Acad. Sci. USA 2011, 108, 11063-11068; (c) J. Talkish, G. May, Y. Lin, J. L. Woolford, C. J. McManus, RNA 2014, 20, 713-720; (d) Y. Ding, Y. Tang, C. K. Kwok, Y. Zhang, P. C. Bevilacqua, S. M. Assmann, Nature 2014, 505, 696-700; (e) C. K. Kwok, Y. Ding, Y. Tang, S. M. Assmann, P. C. Bevilacqua, Nat. Commun. 2013, 4, 2971; (f) R. C. Spitale, R. A. 
Flynn, Q. C. Zhang, P. Crisalli, B. Lee, J.-W. Jung, H. Y. Kuchelmeister P. J. Batista, E. A. Torre, E. T. Kool, H. Y. Chang, Nature 2015, 519, 486490; (g) R. C. Spitale, P. Crisalli, R. A. Flynn, E. A. Torre, E. T. Kool, H. Y. Chang, Nat. Chem. Biol. 2013, 9, 18-20; (h) L. J. Kielpinski, J. Vinther, Nucleic Acids Res. 2014, 42, e70.

[14] A. D. Bolzán, M. S. Bianchi, Mutat. Res.-Rev. Mutat. 2002, 512, 121-134.

[15] D. Gillingham, S. Geigle, O. A. von Lilienfeld, Chem. Soc. Rev. 2016, 45, 2637-2655.

[16] D. T. Beranek, Mutat. Res. 1990, 231, 11-30.

[17] E. Kühnel, D. D. P. Laffan, G. C. Lloyd-Jones, T. Martínez del Campo, I. R. Shepperson, J. L. Slaughter, Angew. Chem. Int. Ed. 2007, 46, 70757078.
[18] A. M. Maxam, W. Gilbert, Proc. Natl. Acad. Sci. USA 1977, 74, 560-564

[19] A. Dobin, C. A. Davis, F. Schlesinger, J. Drenkow, C. Zaleski, S. Jha, P Batut, M. Chaisson, T. R. Gingeras, Bioinformatics 2013, 29, 15-21.

[20] D. T. Beranek, C. C. Weis, D. H. Swenson, Carcinogenesis 1980, 1, 595606.

[21] K. E. Watters, T. R. Abbott, J. B. Lucks, Nucleic Acids Res. 2016, 44, e12.

[22] N. Fei, B. Sauter, D. Gillingham, Chem. Commun. 2016, 52, 7501-7504

[23] J. C. González-Olvera, J. Martínez-Reyes, E. González-Jasso, R. C. Pless, Biophys. Chem. 2015, 206, 58-65. 
Entry for the Table of Contents (Please choose one layout)

Layout 1:

\section{COMMUNICATION}

Text for Table of Contents

Author(s), Corresponding Author(s)*

Page No. - Page No.

Title

((Insert TOC Graphic here))

Layout 2:

\section{COMMUNICATION}

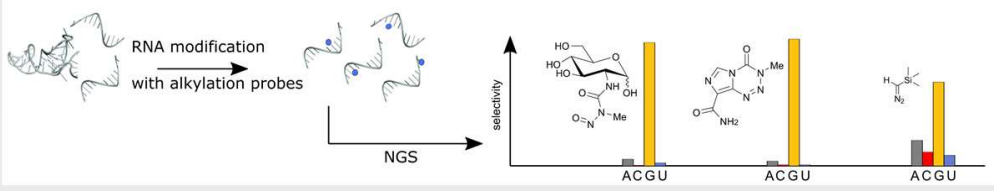

Basilius Sauter, Dennis Gillingham*

Page No. - Page No.

Title

Drugs that covalently modify DNA are components of most chemotherapy regimens, often serving as first-line treatments. Classically, the reactivity and selectivity of DNA alkylating agents has been determined in vitro with short oligonucleotides. Here we use next generation sequencing to report on the chemoselectivity of alkylating agents on RNA and apply this technology to the previously uncharacterized alkylating agent trimethylsilyl diazomethane. 